\title{
Method to Study Water Diffusion into Polymers ${ }^{\dagger}$
}

\author{
Jamila Boudaden ${ }^{1,2, *}$, Matthias Steinmaß1 ${ }^{1}$, Hanns-Erik Endres ${ }^{1}$ and Peter Müller-Buschbaum ${ }^{3}$ \\ 1 Fraunhofer EMFT, Research Institution for Microsystems and Solid State Technologies EMFT, \\ Hansastraße 27d, D-80686 Munich, Germany; matthias.steinmassl@emft.fraunhofer.de (M.S.); \\ hanns-erik.endres@emft.fraunhofer.de (H.-E.E.) \\ 2 Institute of Electronic and Sensor Materials, Technische Universität Bergakademie Freiberg, \\ Gustav-Zeuner-Str. 3, D-09599 Freiberg, Germany \\ 3 Physik-Department, Lehrstuhl für Funktionelle Materialien, Technische Universität München, \\ James-Franck-Strasse 1, D-85748 Garching, Germany; muellerb@ph.tum.de \\ * Correspondence: jamila.boudaden@emft.fraunhofer.de; Tel.: +49-89-54759-161 \\ + Presented at the Eurosensors 2018 Conference, Graz, Austria, 9-12 September 2018.
}

Published: 30 November 2018

\begin{abstract}
We designed and realized a low cost relative humidity (RH) capacitive sensor having an interdigitated transducer coated with a cheap sensing material working at room temperature (polyimide). Thermally perturbed diffusion of water molecules into the polyimide layer is studied by heating the sensor locally and measuring the sensor capacitance change. The swelling and deswelling model is applied to determine the time constant of involved processes. This simple method, using an evaluation kit developed by our group, to measure the sensor capacitance and to study the diffusion process of water molecules into polyimide could be generalized to analyze the gas diffusion processes into polymer based sensing layer generally used in the field of gas chemical sensors.
\end{abstract}

Keywords: polyimide; relative humidity sensor; interdigitated transducer IDT's; thermal stress; thermoresponsive polymer

\section{Introduction}

Polyimide material is intensively used to sense the moisture content in an environment. A polyimide based capacitive humidity sensor measures a change in electrical capacitance of the interdigitated transducer coated with a thin layer of polyimide. The humidity has an effect on a dielectric constant of polymer substance once the water molecule is adsorbed in the sensing layer. The change in the dielectric constant is continuously monitored by measuring the capacitance, which is directly related to the relative humidity. The electrical properties of the polyimide are stable if operated at room temperature, which is the case for humidity sensors. Although, the polyimide consists of cross-linked polymer networks, which is own good thermal, chemical resistance and mechanical strength properties. Those properties are strongly affected by water sorption. Heating the polyimide layer to higher temperature in the presence of humidity show a behavior similar to hydrogel materials. Although, polyimide is probably among the most studied polymers for use in the field of humidity sensors and CMOS technology. The thermosensitivity of polyimides was never reported before. The network structures of the cross-linked polyimide possess the ability to respond to temperature change by undergoing reversible structural rearrangement.

The goal of this work is to investigate the thermoresponsive properties of polyimide using an interdigitated transducer and low power miniaturized system able to monitor the capacitance change and to heat the sensor locally. This method is considered as an inexpensive alternative method that could be extended to other thermoresponsive polymeric materials. 


\section{Experimental Methods}

\subsection{Transducer and Sensor Fabrication}

A pair of interdigitated electrodes, IDE, was used as transducer for relative humidity sensor, socalled Interdigitated transducer, IDT. A heater and the temperature sensor were patterned around the IDE by photolithography technique using the desired mask. Each chip has an area of $2 \times 2 \mathrm{~mm}, 6$ $\mu \mathrm{m}$ finger width, $2 \mathrm{~mm}$ electrode length, and $12 \mu \mathrm{m}$ pitch [1]. The photosensitive polyimide material was spin coated homogeneously on top of the planar electrodes on the 6-inch glass wafer to obtain different layer thicknesses ranging from $1 \mu \mathrm{m}$ to $11 \mu \mathrm{m}$. After completing the photolithographic process, the film was cured in a Yes Oven under N2 inert atmosphere at $400{ }^{\circ} \mathrm{C}$. After dicing the $6 "$ wafer, a single chip, named Interdigitated Transducer IDT, was glued on an adapted printed circuit board (PCB) and wire bonded thereto. The PCB was equipped with zero insertion force (ZIF) connectors.

\subsection{Polyimide Sensor Characterization}

In this study, the gas mixing apparatus was able to generate a constant level of relative humidity by selecting the adopted ratio between the volumes of humid gas to the $\mathrm{N}_{2}$ carrier gas [2].

An evaluation kit, having low power, low cost, compact size, and network compatibility, was designed for high-speed data acquisition (each $150 \mathrm{~ms}$ ). The integrated microcontroller STM32L476RG possesses a several Analog-to-Digital converters (ADC) and a touch sensing controller for capacitive measurements, via the charge transfer method on board. An extension circuit board to simultaneously measure the capacitance of three sensors was designed and realized [1]. The typical sampling time used in this study is $200 \mathrm{~ms}$. The sensors can be heated up to $200{ }^{\circ} \mathrm{C}$ using the heating wire around the sensor interdigitated electrodes. The layer thickness was manually measured by an optical reflectometer. The variation of the polymer layer thickness in contact with humidity as an analyte, gives an idea about the swelling/deswelling of the polymeric layer.

\section{Experiments and Results}

The water uptake of the polyimide based capacitive sensor at temperatures higher that $25{ }^{\circ} \mathrm{C}$ is an important parameter to be investigated. A standard EMFT humidity sensor was used, which consists of a $12 \mu \mathrm{m}$ pitch gold IDT with a polyimide layer of $4.6 \mu \mathrm{m}$ thickness.

The response of the polyimide layer to a temperature change was investigated. At a constant level of humidity $40 \%$, the capacitance change and layer thickness dependent on time were recorded while a thermal stress was applied to the sensor. The thermal stress was reached by heating the sensor from RT to $75^{\circ} \mathrm{C}$. Figure 1 shows the recorded capacitance, the measured polyimide thickness and the reached temperature on the sensor dependent time under thermal stress. The applied thermal stress is obtained by heating the sensors locally from RT to $75^{\circ} \mathrm{C}$. A rectangular voltage pulse (max. $5 \mathrm{~V}$ ) was applied to the heating wire of the sensor insert into the ZIF sockets of the MCU system. Both the capacitance of the IDT and the resistance of the temperature sensor surrounding it were continuously recorded each $200 \mathrm{~ms}$ by the MCU system. It worth to underline that the polyimide layer thickness was measured manually (not in automatic mode) but not in real time.

The measured variation of the polyimide thickness and the capacitance follow the same tendency after applying a thermal stress to the sensor. The response and recovery times $\tau 90$ of the heater wire surrounding the IDT correspond to $20 \mathrm{~s}$. The capacitance shows a fast and sharp decrease by a $0.2 \mathrm{pF}$ during the temperature increase due to the release of water molecule from the polyimide matrix. At the same time, the thickness of the $4.6 \mu \mathrm{m}$ polyimide layer is decreased by $8 \mathrm{~nm}$. After the noticed decrease, the capacitance increases slowly by $0.05 \mathrm{pF}$ to reach a stable value. While observing a slow increase in the capacitance, no remarkable increase in the polyimide layer thickness could be measured. It is assumed that the observed change in the polyimide thickness could not explain the recorded capacitance decrease, as a single influencing parameter. The decrease in the capacitance can 
be correlated either to a change in the refractive index or most probably to a structural rearrangement of the polyimide network.

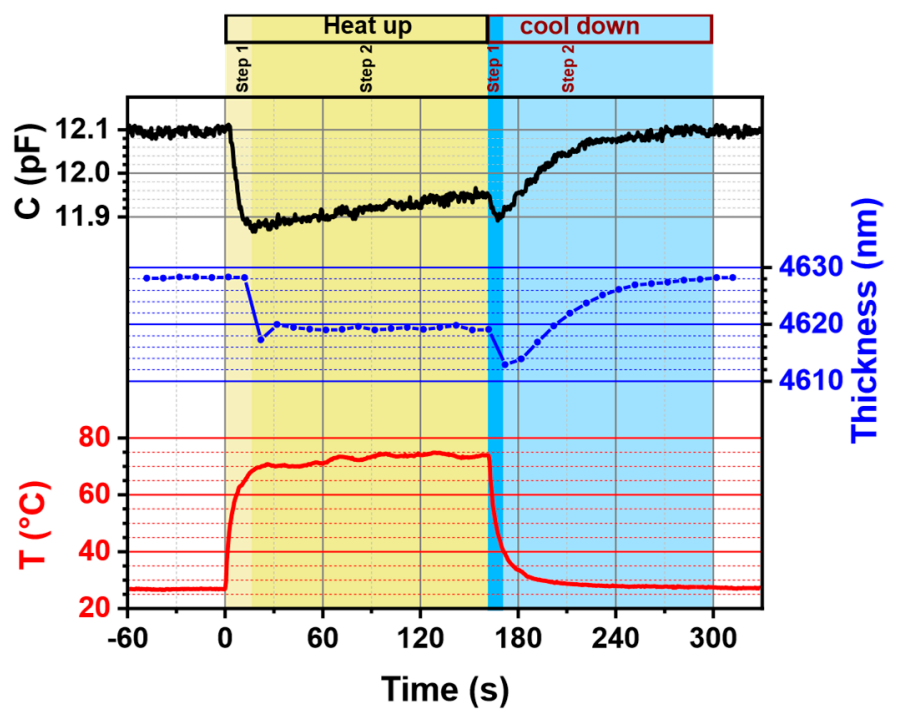

Figure 1. Change of the capacitance and the sensing polyimide layer thickness dependent time after applying a thermal perturbation to the sensor. The thermal perturbation is done by heating up and cooling down the sensor between 27 and $74{ }^{\circ} \mathrm{C}$ for a constant $\mathrm{RH}=40 \%$.

By cooling down the sensor from $74^{\circ} \mathrm{C}$ to $27^{\circ} \mathrm{C}$ within $20 \mathrm{~s}$, the polyimide chains relax and recover back to the original rearrangement. Therefore, the water molecules diffuses into the polymer matrix and the capacitance increases to reach the base capacitance at $27^{\circ} \mathrm{C}$. Zhong et al. studied the swelling and shrinking behavior of hydrogels and reported a similar observation [3]. The diffusion process of water molecule into a heated layer of hydrogels consists of two parts. Each part has its distinct time constant. In the first step, a rapid release of the water is followed by a rearrangement of the polymer network structure. The second increase reflects the water diffusion into the newly formed polymer network. The rearranged network is able to reabsorb water molecules and to attain an equilibrium. The time constants of the processes are obtained using a two-process model for the capacitance based on an exponential diffusion process reported by [4].

$$
C(t)=C_{0}+A_{1}\left(1-\exp \left(-\frac{t-t_{1}}{\tau_{1}}\right)\right)+A_{2}\left(1-\exp \left(-\frac{t-t_{2}}{\tau_{2}}\right)\right)
$$

At time $t_{1}$ a first exponential diffusion process starts and characterized by a diffusion time $\tau_{1}$. At time $t_{2}$ a second process takes place and is characterized by a diffusion time $\tau_{2}$. The diffusion times are reported into the graphs of Figure 2 after applying a thermal stress to a $4.6 \mu \mathrm{m}$ polyimide sensor and after turning off the thermal stress.

Generally, the polyimide shows a transition similar to a phase transition found in Hydrogel material. At elevated temperature, the polymer chains are organized into a new rearrangement. Consequently, the incorporation of water molecules into the polyimide layer cannot be explained by a simple diffusion process, but by a two steps process: polymer collapse and polymer relaxation characterized by two different time constants. 


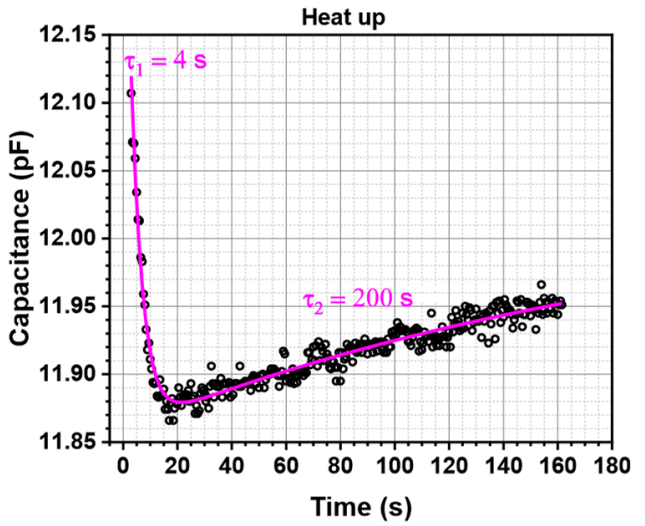

(a)

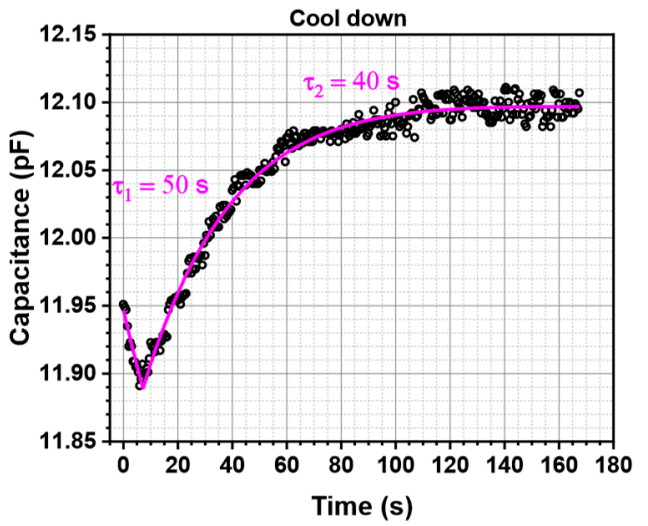

(b)

Figure 2. Diffusion characteristics of water vapor into a heated polyimide layer. The diffusion processes were fitted using Equation from the model of Li and Tanaka [4]: (a) after applying a thermal stress; (b) after turning off the thermal stress.

Author Contributions: J.B. designed the experiments based on previous realized work and headed the research project. M.S. did the experimental work. H.-E.E. and M.S. realized the electronic system "evaluation board", which was financed by the European Commission within the FP7 Strep-ICT- INTERFLEX GA: 247710. P.M.-B. participated actively to discussing the results. J.B. wrote the paper with input from all authors. All authors discussed and commented on the manuscript.

Acknowledgments: The authors wish to thank Andreas Drost, Vu Chi. Hung, R. Faul, and D. Linke for designing and realizing IDTs. The authors wish to thank G.A.M. Nastasi for providing basic concepts of the microcontroller setup. This work was partly financially supported by the Bavarian Ministry of Economic Affairs and Media, Energy and Technology, Germany, within the project "Sensorplattform für Condition Monitoring".

Conflicts of Interest: The authors declare no conflict of interest.

\section{References}

1. Boudaden, J.; Steinmaßl, M.; Endres, H.-E.; Drost, A.; Eisele, I.; Kutter, C.; Müller-Buschbaum, P. PolyimideBased Capacitive Humidity Sensor. Sensors 2018, 18, 1516, doi:10.3390/s18051516.

2. Endres, H.-E.; Jander, H.D.; Göttler, W. A test system for gas sensors. Sens. Actuators B Chem. 1995, 23, 163-172.

3. Zhong, Q.; Metwalli, E.; Kaune, G.; Rawolle, M.; Bivigou-Koumba, A.M.; Laschewsky, A.; Papadakis, C.M.; Cubittd, R.; Müller-Buschbaum, P. Switching kinetics of thin thermo-responsive hydrogel films of poly(monomethoxy-diethyleneglycol-acrylate) probed with in situ neutron reflectivity. Soft Matter 2012, 8, 5241-5249, doi:10.1039/c2sm25401h.

4. Li, Y.; Tanaka, T. Kinetics of swelling and shrinking of gels. J. Chem. Phys. 1990, 92, 1365-1371, doi:10.1063/1.458148.

(C) 2018 by the authors. Licensee MDPI, Basel, Switzerland. This article is an open access article distributed under the terms and conditions of the Creative Commons Attribution (CC BY) license (http://creativecommons.org/licenses/by/4.0/). 